\title{
Susceptible-Infected-Recovered model study using free particle dynamics
}

\author{
F. Garzón ${ }^{a}$, J. Olvera-Orozco ${ }^{b}$, J. Castro-López ${ }^{c}$ and A. Figueroa ${ }^{d}$ \\ ${ }^{a}$ Instituto de Investigación en Ciencias Básicas y Aplicadas, \\ Universidad Autónoma del Estado de Morelos, Cuernavaca, Morelos. \\ ${ }^{b}$ Instituto de Energías Renovables, Universidad Nacional Autónoma de México, Temixco, Morelos. \\ ${ }^{c}$ Universidad del Mar-Campus Puerto Ángel, Oaxaca. \\ ${ }^{d}$ CONACYT-Centro de Investigación en Ciencias, Universidad Autónoma del Estado de Morelos, Cuernavaca, Morelos.
}

Received 20 December 2020; accepted 26 January 2021

\begin{abstract}
A study on the epidemiological Susceptible-Infected-Recovered (SIR) model is presented using free particle dynamics. The study is performed using a computational model consisting of randomly allocated particles in a closed domain which are free to move in random directions with the ability to collide with each other. The transmission rules for the particle-particle interactions are based on the main viral infection mechanisms, resulting in real-time results of the number of susceptible, infected, and recovered particles within a population of $N$ $=200$ particles. The results are qualitatively compared with a differential equation SIR model in terms of the transmission rate $\beta$, recovery rate $\gamma$, and the basic reproductive number $R_{0}$, yielding overall good results. The effect of the particle density $\rho_{p}$ on $R_{0}$ is also studied to analyze how an infectious disease spreads over different types of populations. The versatility of the proposed free-particle-dynamics SIR model allows to simulate different scenarios, such as social distancing, commonly referred to as quarantine, no social distancing measures, and a mixture of the former and the latter. It is found that by implementing early relaxation of social distancing measures before the number of infected particles reaches zero, could lead to subsequent outbreaks such as the particular events observed in different countries due to the ongoing COVID-19 health crisis.
\end{abstract}

Keywords: SIR; free particles; particle density; COVID-19.

PACS: 02.70.-c; 45.50.Tn; 87.15.Aa; 87.19.xd

DOI: https://doi.org/10.31349/RevMexFis.67. 041402

\section{Introduction}

William O. Kermack and Anderson G. McKendrick described the SIR model for the first time in 1927 [1], which was the pioneering model for the study of epidemics using differential equations. There was knowledge regarding the behavior of disease epidemics in those years, and it led to the proposal of a mathematical model which could describe the evolution of arbitrary infections during an epidemic. Kermack and McKendrick proposed a classification of individuals in a population $N$, susceptible $S$, infected $I$, and recovered $R{ }^{i}$ The set of individuals should then behave accordingly with the following system of equations in a certain time period

$$
\begin{aligned}
\frac{d S}{d t} & =-\frac{\beta I S}{N}, \\
\frac{d I}{d t} & =\frac{\beta I S}{N}-\gamma I, \\
\frac{d R}{d t} & =\gamma I,
\end{aligned}
$$

where $S(t), I(t), R(t)$ describe the number of susceptible, infected, and recovered individuals as a function of time, respectively. The recovery and transmission rates are given by $\lambda$ and $\beta$, respectively. The system of non-linear differential (1-3) has been solved algebraically [2] (see Fig. 1), and analytically [3].

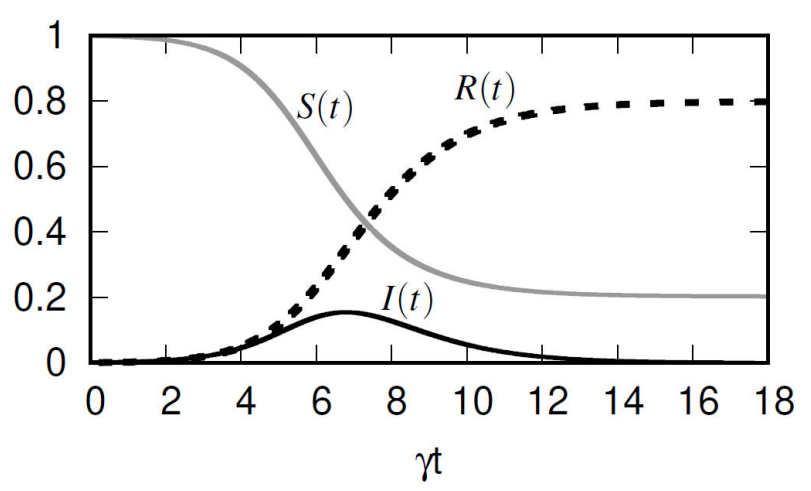

FIGURE 1. Algebraic solution to the system of differential (1-3), assuming non-vital dynamics, where $N=1, S(t)+I(t)+R(t)=$ 1 , with $\beta / \gamma=2, I(0)=10^{-3}, S(0)=1-I(0)$.

Kermack and McKendrick used their model to analyze the data from the 1906 bubonic plague of Bombay and obtained a great quantitative approximation that matched the number of deaths caused by the Yersinia pestis bacterium. The SIR model was used to study the dengue fever breakouts, caused by mosquito bites, of 1997 in Cuba and 2005 in Venezuela [4]. The model was also used to analyze the 1997 outbreak of classical swine fever in the Netherlands, which is a highly contagious viral disease that affects herds of pigs and wild boars [5].

In December 2019, a new coronavirus (CoV) was detected owing to a harsh pneumonia outbreak that is be- 
lieved to have originated in a wet market of Wuhan, China. Later in January 10, 2020, the first genome sequence was made publicly available, revealing a similarity with already known forms of coronaviruses such as the MERS-CoV and more closely to the SARS-CoV. The novel coronavirus was designated severe acute respiratory syndrome coronavirus 2 (SARS-CoV-2) by the Coronavirus Study Group of the International Committee on Taxonomy of Viruses [6]. As of January 20, 2021, according the statistics from the World Health Organization (WHO) [7], there are a total of 94,124,612 confirmed cases and 2,034,527 confirmed deaths due to the SARS-CoV-2.

Scientists from all over the world rushed immediately to find an effective antiviral treatment against the SARS-CoV-2, which is the responsible agent for the ongoing coronavirus disease (COVID-19). The Oxford-AstraZeneca collaboration was the first to publish results from phase III clinical trials of a COVID-19 vaccine on December 8, 2020 [8]. On December 11, 2020, the U. S. Food and Drug Administration (FDA) issued the first emergency use authorization (EUA) for the Pfizer-BioNTech COVID-19 vaccine, and followed with a second EUA for the Moderna COVID-19 vaccine, allowing them to be administered to the public $[9,10]$. Mexico's FDA analogue Comisión Federal para la Protección contra Riesgos Sanitarios (COFEPRIS) also issued an equivalent emergency use authorization on December 11, 2020, for the Pfizer-BioNTech COVID-19 vaccine [11]. On January 4, 2021, the COFEPRIS issued a second emergency use authorization for the AstraZeneca COVID-19 vaccine [12], allowing both companies to distribute their vaccine in the country for their application to the public. Real-time updates and information on the disease and the vaccines can be found summarized in [13]. The collective efforts consist of developing more vaccines, social distancing protocols, and officially imposed quarantines. Additionally, a series of mathematical models have been implemented to estimate the scope and transmission capacity, taking into account numerous factors, of the COVID-19, among them the SIR model [14].

\section{Computational model}

The computational model of this work implements colliding particles that move freely inside a square domain which is never abandoned by the particles. The viral transmission rules which the particles obey are based on the person-toperson spread mechanism, that is, direct and indirect contact as sneezing, coughing, and touching contaminated surfaces.

\subsection{Number of particles, $\mathrm{N}$, and the collision detection method}

A determined number $N$ of particles $i$ with a specific mass are allocated inside a square domain of area $\Omega=l_{x} \times l_{y}$. The intrinsic RANDOM_NUMBER subroutine from Fortran 90 is called to assign each particle a random position $\vec{r}_{i}=\left(x_{i}, y_{i}\right)$ and velocity $\vec{u}_{i}=\left(u_{i}, v_{i}\right)$ as initial conditions over the domain, such that initial positions assume a value within the ranges $0<x_{i}<l_{x}$ and $0<y_{i}<l_{y}$, and initial velocities assume a random value such that $\left\|\vec{u}_{i}\right\|=U$, where $U$ is a constant.

The numerical time integration for the position and velocity of the particles was tested using the Adam-Bashforth, Runge-Kutta, Euler, and Verlet methods. The Verlet method of order $\mathcal{O}\left(\Delta t^{4}\right)$ was ultimately selected owing to its high accuracy and low computational cost, and was developed on a prediction-correction algorithm [15] described as

$$
\begin{aligned}
\vec{r}_{i}^{(n+1 / 2)} & =\vec{r}_{i}^{(n)}+\frac{\Delta t}{2} \vec{u}_{i}^{(n)}, \\
\vec{u}_{i}^{(n+1)} & =\vec{u}_{i}^{(n)}+\Delta t \vec{a}_{i}^{(n+1 / 2)}, \\
\vec{r}_{i}^{(n+1)} & =\vec{r}_{i}^{(n+1 / 2)}+\frac{\Delta t}{2} \vec{u}_{i}^{(n+1)},
\end{aligned}
$$

where the superscript $n$ represents the time which is being calculated. The time step $\Delta t=1 \times 10^{-4} \mathrm{~s}$ is a constant, and $\vec{a}_{i}$ represents externally induced accelerations due to forces such as particle-particle and particle-wall collisions, which were both considered in the present work. The particle-wall collision is a reflective boundary condition, where the direction of the velocity component parallel to the outwardpointing normal of the wall is inverted when the position $\vec{r}_{i}$ of the particle is greater or lower than the dimensions of the domain $\Omega$. This is based on a particular case of boundary conditions of smoothed particle hydrodynamics (SPH) simulations [16]. The particle-particle collision was implemented using undamped normal contact forces [17] resulting from accelerations described as

$$
\vec{a}_{i}^{(n+1 / 2)}=\frac{1}{m_{i}} \sum_{j} \kappa \delta_{i j}^{(n+1 / 2)} \vec{n}_{i j}^{(n+1 / 2)},
$$

where

$$
\delta_{i j}^{(n+1 / 2)}=2 d-\left(\vec{r}_{i}^{(n+1 / 2)}-\vec{r}_{j}^{(n+1 / 2)}\right) \cdot \vec{n}_{i j}^{(n+1 / 2)} .
$$

In Eq. (7), the sum is performed over all neighboring particles $j, m_{i}=1 \mathrm{~kg}$ for all $i$ particles, $\kappa=50 \mathrm{~N} / \mathrm{m}$ is the spring constant, $\vec{n}_{i j}$ is the normal vector between particles $i$ and $j$, and $d=0.01 \mathrm{~m}$ is the contact radius. The collision detection was implemented using a double loop, one for $i$, and a nested one for $j$. Computing the distance between the particles and using equation (8) for the case where $\delta_{i j}>0$, results in longer computation times $\sim \mathcal{O}\left(N^{2}\right)$ when compared against other methods such as the Linked Cell method [17].

\subsection{Particle classification and transmission rules}

In a broad manner, the Kermack-McKendrick model explains mathematically how a epidemic disease spreads amongst a determined number of individuals of a population with certain characteristics, and how its effects come to an end after a time period. In this work, the characteristics of the individuals are narrowed to susceptible $S$, infected $I$, and recovered $R$. Demographic events such as deaths, births, immigration and emigration are not considered. 


\section{Free-for-all$$
\mathrm{N}=200, \mathrm{U}=0.05 \mathrm{~m} / \mathrm{s}, \mathrm{p}_{\mathrm{c}}=0.4
$$

$\begin{array}{rr}\text { Susceptible } & \bullet \\ \text { Infected } & * \\ \text { Recovered } & \bullet\end{array}$

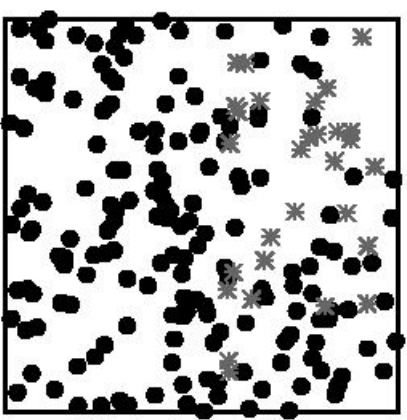

$\mathrm{t}=13.30$

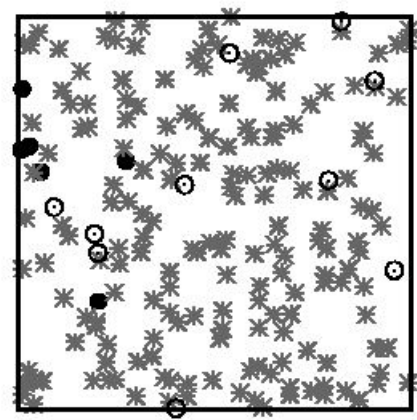

$\mathrm{t}=33.25$

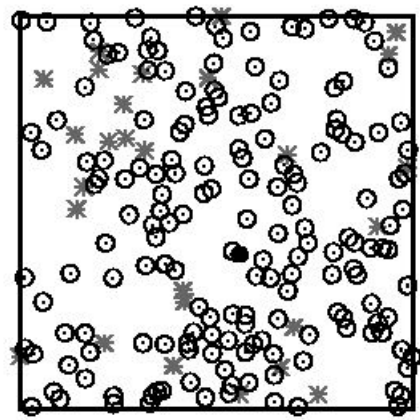

$\mathrm{t}=53.20$

FIGURE 2. All susceptible particles $(\bullet)$ move freely. At $t=0.0$ an infected particle $(*)$ is introduced and begins the infection process, i.e., the start of the pandemic. An exponential growth is observed at $t=6.65 \mathrm{~s}$ and $t=19.95 \mathrm{~s}$. At $t=26.60 \mathrm{~s}$ the first recovered particles $(\circ)$ are observed. Later, an exponential decay of infected particles occurs at $t=33.25$ and $t=29.90 \mathrm{~s}$. Finally, the number of recovered particles is predominant.

In our model, each case begins with all particles classified as susceptible, except for one randomly infected particle $I_{z}$ (patient zero) with the capacity to alter the state of every particle it encounters. Naturally, every new infected particle now has the capacity to also infect susceptible particles. In order to yield results that can resemble a real-life scenario with more accuracy, the at-contact infection mechanism is replaced by an infection mechanism based on a probability parameter $p_{c}$ and is implemented as follows. All infected par- ticles will be surrounded by an infection radius $R_{c}=0.03 \mathrm{~m}$ with a known $p_{c}$, when a susceptible particle approaches an infected one close enough to be inside $R_{c}$, a randomly assigned value is given to a variable $r n d$. If $r n d>p_{c}$ the state of the susceptible particle remains unchanged, giving it the possibility of not becoming infected even if it still inside $R_{c}$. On the other hand, if $r n d \leq p_{c}$ the susceptible particle now becomes infected. This procedure is only assigned once to susceptible particles every time they are inside $R_{c}$. An in- 
fected particle changes its state to recovered after exceeding a recovery time $t_{r}=25 \mathrm{~s}$ (infectious period), and a recovered particle cannot become infected again. The recovery time has been considered a constant for all infected particles. In this work the value for $t_{r}$ is irrelevant, although it is subject to further discussion.

\section{Results}

\subsection{Free-for-all scenario}

The initial configuration, referred to as free-for-all scenario, consists of particles created inside a square domain of sides $\Omega=1 \times 1 \mathrm{~m}$, with random position, moving in random directions with a constant velocity magnitude of $U=0.05 \mathrm{~m} / \mathrm{s}$, colliding into each other with a $p_{c}=0.4$ (which remained constant for all configurations). In this scenario, the initial conditions are $S(0)=199$ and $I(0)=1$. The evolution of the position of all particles is depicted in Fig. 2, where the first 9 time frames were captured every $6.65 \mathrm{~s}$ for a maximum time, $t_{\max }$, of $120 \mathrm{~s}$. When an infected particle changes the

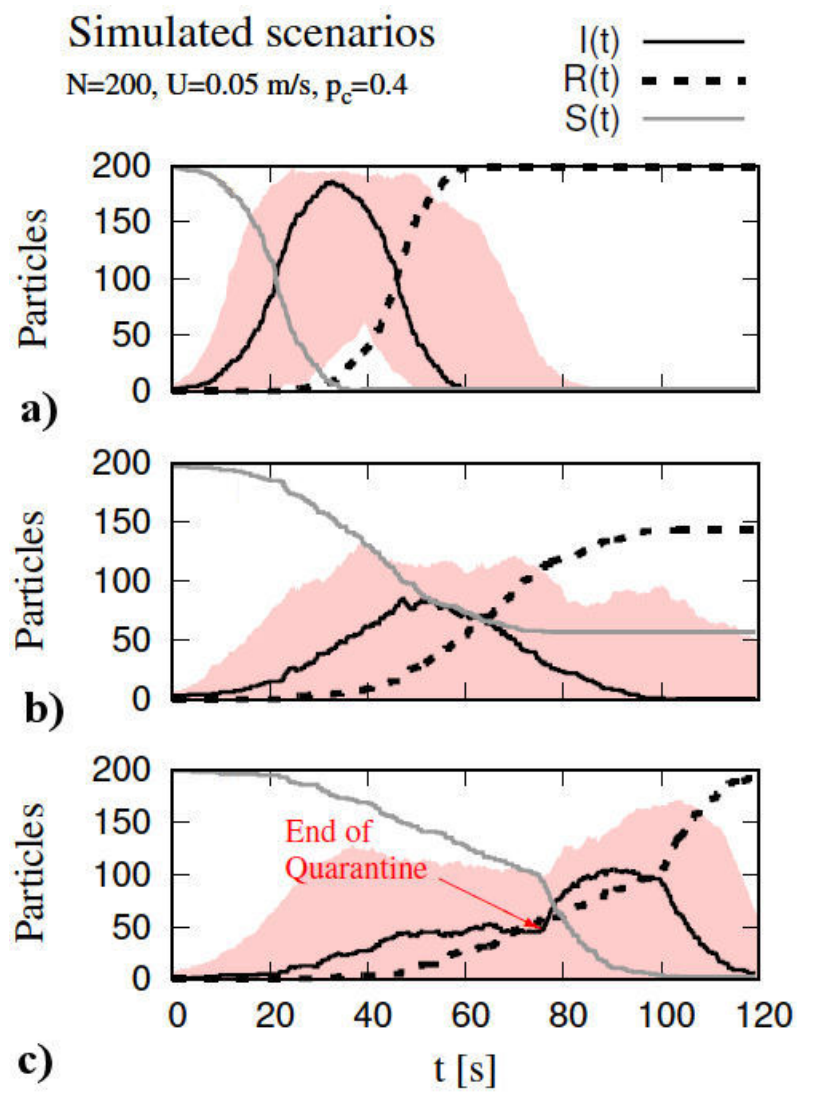

FIGURE 3. Projection curves where susceptibles are solid gray line, infected are solid black line, and recovered are dashed black line. a) free-for-all, b) quarantine, and c) end-of-quarantine. The number of infected particles for each scenario is inside the red background region, which is the result of 400 additional simulations. In all cases, the ordinate shows the number of particles, whereas the abscissa represents time. state of a susceptible particle to infected, the latter can change the state of other susceptible particles while it remains within the recovery time, consequently generating an exponential change of susceptible to infected particles (see Fig. 3a)). The maximum number of infected particles, $I_{\max }$, was recorded at $t=32.3 \mathrm{~s}$ for $I_{\max }=186$, representing $93 \%$ of the total population. Following $I_{\max }$, an exponential decay of infected particles is recorded along with an increase in the number of recovered particles. Eventually, the entire population shifted from susceptible to infected, and finally all recovered at the end of the simulation.

\subsection{Quarantine scenario}

The second configuration, referred to as quarantine, begins by randomly distributing the particles inside the domain and fixing $80 \%$ of the particles as immovable regardless of collisions and changes of state. The remaining $20 \%$ of particles have the same constant velocity $U=0.05 \mathrm{~m} / \mathrm{s}$ and move in random directions with $I_{z}$ included in this percentage. The stationary particles can change the direction of moving particles when they interact, and they can also become infected (see Fig. 3b)). A similar behavior to that of the free-forall scenario was observed, that is, an exponentially growing number of infections which eventually decays. The maximum number of infected particles, $I_{\max }$, was recorded at $t=47.3 \mathrm{~s}$ for $I_{\max }=85$, representing $42.5 \%$ of the total population. At the end of the simulation, a total of 143 particles shifted from infected to recovered, representing $71.5 \%$ of the population; whereas 57 particles kept their initial susceptible state, representing $28.5 \%$ of the population.

\subsection{End-of-quarantine scenario}

The third configuration is referred to as end-of-quarantine owing to its inherited configuration from the quarantine scenario. This configuration begins identical to the quarantine scenario, until a $t_{0}=75 \mathrm{~s}$ is reached, and at this point, all particles are free to move, recovering the free-for-all scenario. Prior to $t_{0}$, a very similar behavior to the quarantine scenario was observed. After $t_{0}$, a new exponential growth of infected cases was recorded (see Fig. 3c)). The maximum number of infected particles $I_{\max }$ was recorded at $t=89.9 \mathrm{~s}$ for $I_{\max }=105$, representing $52.5 \%$ of the total population. At the end of the simulation, a total of 193 particles shifted from infected to recovered, representing $96.5 \%$ of the population; whereas only 2 particles kept their initial susceptible state and 5 particles remained infected [4].

Every scenario was simulated 400 times with different seeds, i.e., the function RANDOM_SEED was used for each new case while maintaining the parameters used for the previous scenarios as constant. For each scenario, Fig. 3 shows in red, the region where the results were obtained for the number of particles $(I)$ according to the 400 simulated cases. In the free-for-all scenario (Fig. 3a)), we obtained a maximum from the set of all maxima belonging to each case 
of infected particles $(I)$ as $\left(I_{\max }\right)_{\max }=197$ representing $98.5 \%$ of all particles. In the quarantine scenario (Fig. 3b), $\left(I_{\max }\right)_{\max }=131$, representing $65.5 \%$ of the total. Finally, in the end-of-quarantine scenario, (Fig. 3c), $\left(I_{\max }\right)_{\max }=171$ which represents $85.5 \%$ of the total.

\subsection{Transmission rate $\beta$, recovery rate $\gamma$, and basic re- production number $R_{0}$}

An analysis was performed on the recovery rate $\gamma$, the transmission rate $\beta$, and the basic reproduction number $R_{0}$ for all
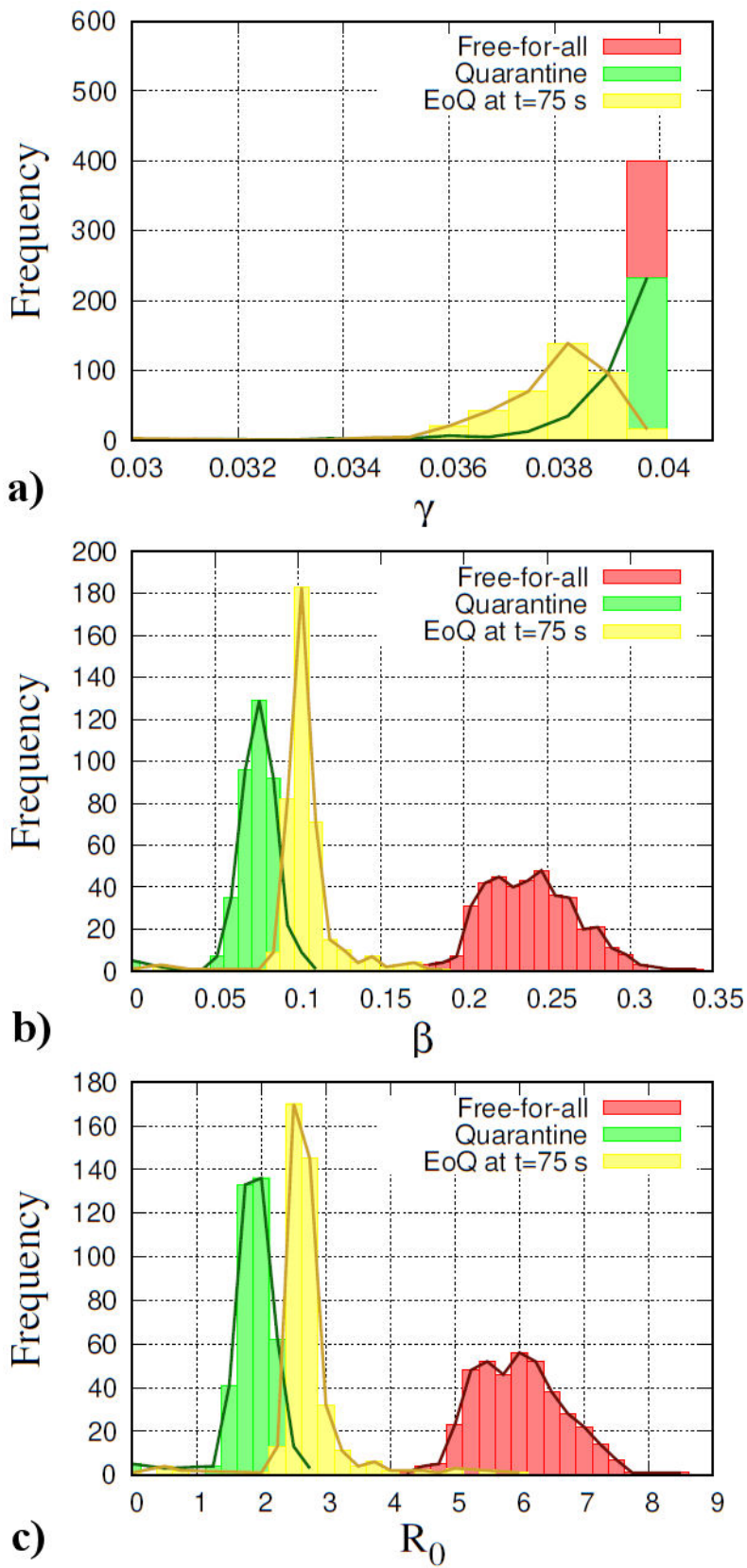

FIGURE 4. a) recovery rate frequency $\gamma_{m}$ with a sampling of $\delta \gamma=0.00075, \mathrm{~b})$ transmission rate $\beta_{m}$ with $\delta \beta=0.0085$, and c) basic reproduction number $\left(R_{0}\right)_{m}$ with $\delta R_{0}=0.25$, for all $m=1,2,3, \ldots, 400$ simulated scenarios. three scenarios. Initially, we studied the area under the curve for the set of data describing the number of particles $(I)$, and the function that is obtained by multiplying the data of $(I)$ by $(S)$, these are $A(I)$ and $A(I S)$, where $A$ represent the area under the curve computed using Simpson's $1 / 3$ method of numerical integration

$$
A_{m}(f)=\sum_{n} \frac{1}{3} h\left(f_{n-1}+4 f_{n}+f_{n+1}\right),
$$

where $h=500 \Delta t$, and $n=1,3,5,7 \ldots$ is the index representing the data values contained in all simulated cases $m=1,2, \ldots, 400$. Afterwards, Eqs. (1) and (3) from the Kermack-McKendrick model were used to study the behavior of the rates described by epidemiology, which, in their integral form, constitute the following identities

$$
\begin{aligned}
& \beta_{m}=-N \frac{S_{m}\left(t_{\max }\right)-S_{m}(0)}{A_{m}(I S)}, \\
& \gamma_{m}=\frac{R_{m}\left(t_{\max }\right)-R_{m}(0)}{A_{m}(I)},
\end{aligned}
$$

where all cases $m$ considered the full set of data from a time $t=0$ to $t=t_{\max }$. In our model, the recovery rate $\gamma_{m}$ represents the amount of new particles $(R)$ over a unit of time over the number of susceptible particles within the population (see $4 \mathrm{a})$ ). The recovery rate $\beta_{m}$ is the ratio of particle-particle contact multiplied by the probability parameter $p_{c}$ (see Fig. $4 \mathrm{~b}$ )). The basic reproductive number $\left(R_{0}\right)_{m}=\beta_{m} / \gamma_{m}$ is defined as the number of new infected particles within the infectious period of any arbitrary infected particle $^{i i}$ (see Fig. 4c)).

\subsection{Basic reproductive number $R_{0}$ as a function of par- ticle density}

The behavior of the basic reproductive number $R_{0}$ was analyzed as a function of the particles $N$ (see Fig. 5). The domain size $\Omega$ was maintained constant with $1 \times 1 \mathrm{~m}$ in order to achieve a proportional particle density $\rho_{p}=N / \Omega$ to the total number of particles. It was noticed that $R_{0}$ increases monotonically for the free-for-all and quarantine scenarios. In the latter and former scenarios, the mean free path is shortened due to the high particle density, consequently increasing the ratio of particle-particle contacts as well as the transmission rate, thus validating the relation $R_{0} \propto \beta$. Regarding endof-quarantine scenario for time $t=75 \mathrm{~s}$, a non-monotic behavior is observed, as can be seen from the inflection point at $N=178$. For all cases, $R_{0}>1$, except for the quarantine scenario, at $N=75, R_{0}=0.8217$. It is important to highlight that $R_{0}$ for end-of-quarantine scenario is very similar to quarantine scenario for particle densities greater than $250 / \mathrm{m}^{2}$.

\section{Conclusions}

In Fig. 3a), a monotonically decreasing function is represented, where the number of susceptible particles declines 


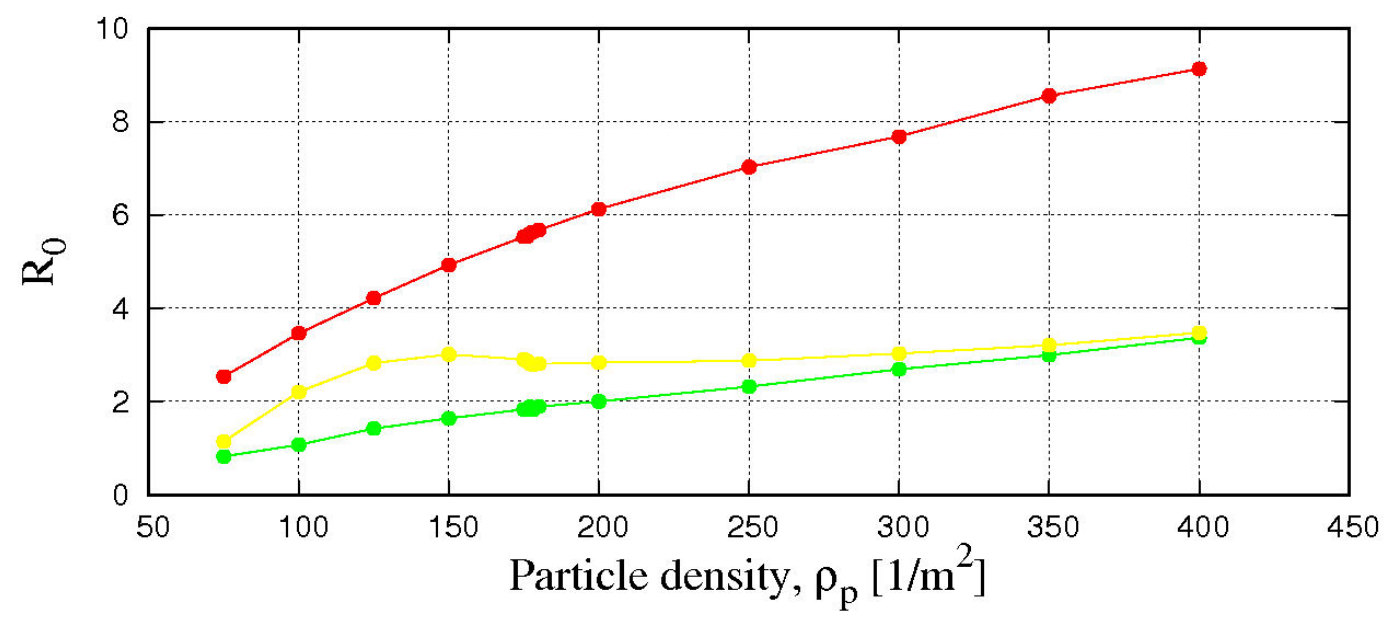

FIGURE 5. Basic reproductive number $R_{0}$ behavior as a function of the number of particles $N$ with a constant domain size of $1 \times 1$ m. Free-for-all (Red), Quarantine (Green) and End-of-quarantine (Yellow).

with respect to time, therefore, its change in time is negative, $S^{\prime}(t)<0$. In turn, the number of recovered particles increases with respect to time, i.e., it is represented by a monotonically increasing function, making its change in time positive, $R^{\prime}(t)>0$. On the other hand, the number of infected particles is non-monotonous and resembles a gaussian distribution, having only one increasing region and one decreasing region. Besides, the inverse of the recovery rate, described by (11), is in qualitative agreement with the recovery time $t_{r}=25 \mathrm{~s}$. This can be seen in Fig. $4 \mathrm{a}$ ), where the free-for-all histogram shows that all cases reported a recovery rate close to $\gamma_{m}=0.04$, making $1 / \gamma_{m}=25$. These results are in qualitative agreement with the description from the Kermack-McKendrick model (Fig. 1). The characteristic monotonic behavior of the curves in the quarantine scenario seems to be conserved, showing certain similitude with the Kermack-McKendrick model, however, limiting the movement of a great number of particles greatly affects the ratio of particle-particle contacts, which consequently influences the transmission rate and the basic reproductive number, respectively (see Figs. 4b) and 4c), where the arithmetic mean of the distribution of the quarantine scenario, $(\bar{\beta} \simeq 0.07892$, $\left.\bar{R}_{0} \simeq 2.0043\right)$, is lower than that for the free-for-all scenario, ( $\bar{\beta} \simeq 0.24491, \bar{R}_{0} \simeq 6.1287$ ). Besides, the maximum number of infected particles for all 400 cases is $33 \%$ lower than in the free-for-all scenario.

Unlike the differential equation model, our model is able to modify the behavior of the particles to simulate ending a quarantine before the number of particles $(I)$ reaches zero. This was hypothesized in order to study the effects of relaxing social distancing measures just before planned. Prior to $t=75 \mathrm{~s}$, the results are similar to the quarantine scenario, after the number of particles $(I)$ varies, we observe for most cases two infection stages corresponding to two maxima in the number of particles $(I)$. In some cases, the second growth stage is significantly larger than the first one, reaching a maximum of 171 particles $(I)(85.5 \%)$. As shown in Fig. 4c) the mean value of the basic reproductive number distribution ( $\bar{R}_{0} \simeq 2.8329$ ), is slightly superior to that of the quarantine event. In turn, ending a quarantine event before the number of infected cases reaches zero, could lead to more violent outbreak, and consequently, to a collapse of the public health system of the population. This type of outbreak was observed in Israel, where on June 1, 2020, the WHO reported an excessive increase in the number SARS-CoV-2 positive cases after the first stage of infections [18]. Scientists attribute this phenomenon to the reopening of the economy and schools. An abrupt outbreak was documented in Mexico on September 2020, approximately three months transpired of a somewhat stable period of low daily confirmed cases, later on November 24 of the same year, 9,187 new cases were confirmed. This was followed by a continuous increase of daily confirmed cases of approximately 50\% with respect to the first ten months of the year, which is now regarded as the second stage of infections in the country [19]. Several speculations can be made regarding the cause of this outbreak, among them, the relaxation of social distancing measures, though no official reasons can be attributed. The cumulative daily cases of both mentioned countries match very closely the qualitative behavior of an outbreak described in our model, particularly for an end-of-quarantine scenario.

In countries whose population density is relatively low such as New Zealand and Australia, the basic reproductive number $R_{0}$ of infectious diseases can be small when compared to that of countries with high population density such as Mexico, U.S.A, and China (refer to Fig. 5). This can be better understood by looking at the number of confirmed cases and deaths from COVID-19 as of October 16, 2020, for New Zealand and China [20,21].

Our particle model is a perfect disease transport model, contrary to local transmission models such as the cellular automata in [22]. Nevertheless, the qualitative results, presented in the latter and the current work, regarding the transmission rate, are very similar under a perfect mixing scenario 
such as the free-for-all. This is justifiable due to the strong relation between the transmission rate, the particle-particle contact rate, and the infective probability, which in the cellular automata model, the cell-cell contact ratio is constant, in turn only modifying the infective probability parameter. On the other hand, our model allows us to keep the probability parameter as a constant, while being able to modify the particle-particle contact ratio when the particle density is increased or decreased.

\section{Discussion, observations, and notes to the reader}

- If the contact radius $d$ is larger than the infection radius $R_{c}$ for all particles, then the infection rule is not satisfied and it is not possible to observe an overall evolution of the system. That is, the infected particle $I_{z}$ cannot change the state of susceptible (S) particles to infected (I), (see Sec. 2.2). This scenario is a perfect analogy to that of physical distancing in real life. A numerical implementation of a randomized contact radius could very well replicate a real life physical distancing behavior.

- In epidemiological theory, the infectious period of an infected individual lasts until recovery or death (recovery time $t_{r}$ ), which is not the same for all individuals, however, for the sake of simplicity, we take the average $t_{r}$ of a certain population.

- The infection radius $R_{c}$ estimates the radial distance at which an infected individual $(I)$ can emit particles with pathogens by sneezing or coughing, which are, at some point, indirectly ingested or inhaled by a susceptible individual $(S)$. This phenomenon was reported as one the main transmission modes of infectious dis- eases such as the common flu, SARS-CoV or SARSCoV-2 [23].

- The probability parameter $p_{c}$ is related to the hygienic actions of a susceptible individual, such as hand washing [24], or the use of sanitary personal protective equipment (PPE) like face masks or gloves [25]. In principle, this parameter is unique of each individual in a population.

- Obtaining real results implies great computational complexity, such as increasing the number of particles $N$ to thousands $\left(\sim \mathcal{O}\left(10^{3}\right)\right)$ or millions $\left(\sim \mathcal{O}\left(10^{6}\right)\right)$, which represents larger computation times. This would also require simulations that resemble the behavior of real-life individuals in a social environment, and not as free particles.

- A study on the effect of population density in infectious disease models [26] reveals that, for extremely dense populations, the saturation of individuals limits their ability to make come in contact with each other, thus decreasing the infection rate. We consider that our results do not contemplate particle saturation until $N=400$ given that $R_{0}$ is proportional to $\beta$.

\section{Acknowledgements}

The authors would like to thank the members of the Laboratorio de Medios Continuos of the Centro de Investigacion en Ciencias at UAEM, for their feedback and observations regarding the development of the SIR model presented here.

F. Garzón and J. Olvera-Orozco acknowledge a grant from CONACYT (México). A. Figueroa thanks the Cátedras CONACYT program. This research was supported by CONACYT under project 258623. $i$. Kermack and McKendrick designated a permanently immune or death individual as "removed".

ii. In practice measured from the patient zero $I_{z}$.

1. W. O. Kermack and A. G. McKendrick, A contribution to the mathematical theory of epidemics, Proc. R. Soc. Lond A 115 (1927) 700, https://doi.org/10.1098/rspa. 1927.0118

2. A. M. Carvalho and S. Gonçalves, An Algebraic Solution for the Kermack- McKendrick Model, arXiv:1609.09313.

3. M. Bohner, S. Streipert, and D. F. M. Torres, Exact solution to a dynamic SIR model, Nonlinear Anal. Hybrid Syst. 32 (2019) 228, https://doi.org/10.1016/j.nahs. 2018.12 .005

4. J. L. Hernández Cáceres, Extracting useful information from dengue incidence data, Rev. Cuba. Inf. Méd. 2 (2007).
5. A. Stegeman, A. R. W. Elbers, J. Smak, and M. C. M. de Jong, Quantification of the transmission of classical swine fever virus between herds during the 1997-1998 epidemic in The Netherlands, Prev. Vet. Med. 42 (1999) 219, https://doi.org/ $10.1016 / \mathrm{S} 0167-5877$ (99)00077-X

6. A. E. Gorbalenya et al. (Coronaviridae Study Group of the International Committee on Taxonomy of Viruses), The species Severe acute respiratory syndrome-related coronavirus: classifying 2019-nCov and naming it SARS-CoV-2, Nat. Microbiol. 55 (2020) 536, https://doi.org/10.1038/ s41564-020-0695-z

7. https://www.who.int/emergencies/diseases/ novel-coronavirus-2019

8. M. Voysey et al., Safety and efficacy of the ChAdOx1 nCov-19 vaccine (AZD1222) against SARS-CoV-2: an interim analysis of four randomised controlled trials in Brazil, South Africa, 
and the UK, Lancet 397 (2021) 99, https://doi.org/ $10.1016 /$ S0140-6736(20)32661-1.

9. https://www.fda.gov/news-events/ press-announcements/fda-takes-keyactionfight-against-covid-19-issuing-emergency-use -authorization-first-covid-19

10. https://www.fda.gov/news-events/ press-announcements/fda-takesadditionalaction-fight-against-covid-19-issuingemergency-use-authorization-secondcovid

11. https://www.gob.mx/cofepris/articulos/ la-cofepris-otorga-autorizacionpara-uso-deemergencia-a-vacuna-para-prevenir-laenfermedad-por-coronaviruscovid-19

12. https://www.gob.mx/cofepris/es/articulos/ autorizacion-para-uso-deemergencia-a-vacuna -astrazeneca-covid-19?idiom=es

13. https://www.nytimes.com/interactive/2020/ science/coronavirus-vaccinetracker.html? auth=login-google1tap\&login=google1tap

14. I. Cooper, A. Mondal, and C. G. Antonopoulos, A SIR model assumption for the spread of COVID-19 in different communities, Chaos Solitons Fractals 139 (2020) 110057, https: //doi.org/10.1016/j.chaos.2020.110057.

15. P. O. J. Scherer, Computational Physics, 3rd ed. (SpringerVerlag, Berlin, 2010), p. 312, https://10.1007/ 978-3-642-13990-1

16. C. A. Dugra Fraga Filho, Smoothed Particle Hydrodynamics, 1st ed. (Springer, Switzerland, 2019), pp. 56-60, https : //10.1007/978-3-030-00773-7

17. B. Muth, M.-K. Müller, P. Eberhard, and S. Luding, Collision Detection and Administration Methods for Many Particles with
Different Sizes, in Proceedings of MEI (Minerals Engineering International), Brisbane, 2007.

18. https://covid19.who.int/region/euro/ country/il

19. https://covid19.who.int/region/amro/ country/mx

20. https://covid19.who.int/region/wpro/ country/nz

21. https://covid19.who.int/region/wpro/ country/cn

22. R. Mansilla and J. L. Gutierrez, Deterministic Site Exchange Cellular Automata Models for the Spread of Diseases in $\mathrm{Hu}$ man Settlements, Complex Syst. 13 (2001) 143.

23. T. Yadav and S. K. Saxena, Transmission Cycle of SARS-CoV and SARSCoV- 2, in Coronavirus Disease 2019 (COVID-19), edited by S. K. Saxena, 1st ed. (Springer, Singapore, 2020), https://doi.org/10.1007/978-981-15-4814-7

24. S. A. Ansar, V. S. Springthorpe, S. A. Sattar, W. Tostowaryk, and G. A. Wells, Comparison of cloth, paper, and warm air drying in eliminating viruses and bacteria from washed hands, Am. J. Infect. Control 19 (1991) 243 https ://doi.org/10 . 1016/S0196-6553(05)80256-1.

25. N. J. Rowan and J. G. Laffey, Challenges and solutions for addressing critical shortage of supply chain for personal and protective equipment (PPE) arising from Coronavirus disease (COVID19) pandemic - Case study from the Republic of Ireland, Sci. Total Environ. 725 (2020) 138532, https : / / doi. org/10.1016/j.scitotenv.2020.138532

26. H. Hu, K. Nigmatulina, and P. Eckhoff, The scaling of contact rates with population density for the infectious disease models, Math. Biosci. 244 (2013) 125, https : / / doi.org/10. $1016 / j . m b s .2013 .04 .013$ 